\title{
Inertial and Gravitational Mass Described by Their Electromagnetic Processes
}

\author{
Hans W. Giertz \\ Uppsa Research, Gnesta, Sweden \\ Email: hans@miklagaard.com \\ Received 5 February 2014; revised 25 March 2014; accepted 17 April 2014 \\ Copyright (C) 2014 by authors and Scientific Research Publishing Inc. \\ This work is licensed under the Creative Commons Attribution International License (CC BY). \\ http://creativecommons.org/licenses/by/4.0/ \\ c) (i) Open Access
}

\begin{abstract}
In the present paper, gravitational and inertial mass are described as one and the same electromagnetic mechanism in atoms and particles. Previous reports have described the dynamic electromagnetic processes of the atom, the photon and gravity. Results from these reports have been compiled into a theoretical model. The theoretical model describes the mechanism creating gravitational and inertial mass. The study also displays how the electromagnetic characteristics of gravitational and inertial mass and the mechanism are measured.
\end{abstract}

\section{Keywords}

Gravitational Mass, Inertial Mass, Higgs Field, Higgs Mechanism, Atom, Gravity, Photon

\section{Introduction}

It has been reported that the universe contains a gigantic singularity which generates very low frequency and synchronized TEM (transverse electromagnetic) waves, having extremely high amplitude and energy [1]-[3]. Atoms contain oscillators with different natural frequencies. These oscillators absorb TEM waves at specific frequencies. The absorbed electromagnetic energy accounts for atomic internal and external forces as well as atomic stored energy and emitted energy [1]-[3]. These TEM waves are impossible to measure using state of the art methods. However, a novel method enables exact characterization of TEM waves emitted by the singularity, absorbed by atoms and re-emitted by atoms [1]-[4].

It has also been reported that atoms absorb these TEM waves in the frequency range $0.005-0.03 \mathrm{~Hz}[1]$. The absorbed energy is very large and may account for atomic forces and atomic stored energy.

Furthermore, it has been reported that atoms also absorb TEM waves with frequency $69.9 \mathrm{~Hz}$ [2]. These TEM waves are called gravity waves in the present paper. These gravity waves are then re-emitted uniformly into space, whereby some are absorbed by other atoms. This creates a mutual flow of synchronized TEM waves am- 
ong atoms, resulting in a mutual force of attraction, i.e. gravity.

Gravitational mass is the expression of the mechanism, i.e. intrinsic atomic oscillators, which absorb and re-emit TEM waves resulting in the gravitational force [2].

It has also been reported that excess energy quanta $h v$ in oscillators, contained in a particle, an electron or an atom, is superpositioned on gravity waves absorbed by the oscillator. The re-emitted TEM wave has the superpositioned energy quanta $h v$ and the frequency $69.9 \mathrm{~Hz}+v$ [3]. The superpositioned energy quantum $h v$ is called photon and it explains the photon-wave duality. Empiric results reveal that this process is frequency independent [3] [4].

Consequently, acceleration or deceleration of a particle, an electron or an atom, results in generated and emitted photons. Acceleration or deceleration of a particle, an electron or an atom demands enforced force and energy. The enforced energy equals the emitted photon energy. Thus inertial mass is the expression of the mechanism, i.e. intrinsic atomic oscillators, which superpose excess energy quanta $h v$ on absorbed and re-emitted TEM waves. Thus, inertial mass is the expression of the mechanism which transforms enforced energy into emitted electromagnetic energy.

Hence, gravitational and inertial mass can be described by the electromagnetic interaction between particles, electrons or atoms and omnipresent electromagnetic energy generated by a singularity.

The objective with the present paper is to build on results already presented in reports [1]-[4]. This information is compiled into a theoretical model in Section 2, which describes the mechanism behind gravitational and inertial mass. The theoretical model is based on state of the art electromagnetic and wave theory [5]-[11] and photon theory [12]-[14]. In Section 3 the theoretical model is compared with state of the art description of inertial mass based on the Higgs field, the Higgs mechanism and the Higgs particle [15]-[21]. Discussion and conclusions are found in Section 4.

The aim of the study is to present a theoretical model of gravitational and inertial mass.

\section{Theoretical Model}

\subsection{Gravity}

The theoretical model builds on the observation that atomic oscillators ${ }_{69.9}$, with natural frequency $69.9 \mathrm{~Hz}$, absorb TEM waves with frequency $69.9 \mathrm{~Hz}$ [2]. The TEM waves originate from a singularity in the universe and have been thoroughly described [1]-[3]. The absorbed TEM waves are then re-emitted uniformly into space.

The behavior and characteristics of plane TEM waves is independent of their frequency. The field vectors $\mathbf{E}$ and $\mathbf{B}$ or $\mathbf{H}\left(\mathbf{B}=\mu_{0} \mathbf{H}\right)$ are linked and perpendicular. The field vector amplitudes relate as $E=c B$, where $c$ is the speed of light. The electric and magnetic field vector amplitudes $E$ and $B$ decrease with the distance from the source as $1 / r$. The Poynting vector $\mathbf{S}$ describes the TEM energy flow density [5] [6]

$$
\mathbf{S}=\mathbf{E} \times \mathbf{H} .
$$

The energy stored in the magnetic field is just equal to that in the magnetic field. The direction of energy flow is reversed for a wave travelling in the opposite direction because the phase of $\mathbf{E}$ and $\mathbf{H}$ is reversed.

Assume that one source, Source 1, absorbs and re-emits plane TEM 1 waves, described by its Poynting vector $\mathbf{S}_{1}(r)$ at the distance $r$ from Source 1. Another source, Source 2 is positioned at the distance $r$. Source 2 absorbs TEM waves and re-emits plane $\mathrm{TEM}_{2}$ waves uniformly into space. In the direction towards Source 1 plane $\mathrm{TEM}_{1}$ and $\mathrm{TEM}_{2}$ waves interact because of the force between their synchronized field vectors. This also implies that there is energy transfer from $\mathrm{TEM}_{1}$ to $\mathrm{TEM}_{2}$ when $\mathrm{TEM}_{2}$ propagates towards Source 1 . In equilibrium there is energy transfer in both directions, i.e. from $\mathrm{TEM}_{1}$ to $\mathrm{TEM}_{2}$ vice versa. Note that $\mathbf{E}$ and $\mathbf{H}$ are always perpendicular and hence $\mathbf{S}=\mathbf{E} \times \mathbf{H}$ can be replaced by $S=E \cdot H$. Equilibrium is defined by the amount of energy that Source 1 is able to deliver at Source 2, e.g. at the distance $r, S_{1}(r)$, and the amount of energy that Source 2 is able to deliver at Source 1, i.e. $S_{2}(r)$. This results in back reaction force $F(r)$, i.e. radiation reaction [5]. This creates mutual force of attraction $F(r)$, and where $\gamma$ is a constant:

$$
F(r)=\gamma S_{1}(r) \cdot S_{2}(r) .
$$

It is now assumed that each source consists of many oscillators $_{69.9}$ where each atomic oscillator $_{69.9}$ re-emits TEM waves described by its Poynting vector $\delta S(r)$ and where $r$ denotes the distance from the source and its oscillator $_{69.9 .} \mathbf{E}$ and $\mathbf{H}$ decrease linearly with the distance $r$ implying that $\delta S(r)$ decreases with the square of 
the distance; $\delta S(r)=\delta S(0) / r^{2}$. The mutual force of attraction $\delta F(r)$ between two atomic oscillators ${ }_{69.9}$ and distance $r$ is

$$
\delta F(r)=\gamma \delta S \cdot \delta S / r^{2}
$$

It is now assumed that one oscillator $_{69.9}$ acts on $p$ co-located oscillators 69.9 , belonging to the set $\mathbf{P}$ of all oscillators $_{69.9}$ at Source 2, and where each force can be described by $\delta F(r)$. These oscillators 69.9 are synchronized resulting in the force

$$
\sum_{p \in P} \delta F(r)=\gamma \delta S \sum_{p \in P} \delta S / r^{2}=\gamma(\delta S \cdot \delta S) p / r^{2} .
$$

It is now assumed that $n$ co-located oscillators $_{69.9}$, belonging to the set $\mathbf{N}$ of all oscillators 69.9 at Source 1 , act on $p$ collocated oscillators 69.9 , belonging to the set $\mathbf{P}$ of all oscillators ${ }_{69.9}$ at Source 2, resulting in the total force $F(r)$

$$
F(r)=\gamma \sum_{n \in N} \delta S \cdot \sum_{p \in P} \delta S / r^{2}=\gamma(\delta S \cdot \delta S) n p / r^{2} .
$$

$\delta S \cdot \delta S$ is a constant described by the intrinsic characteristics of the oscillator ${ }_{69.9}$, and hence Equation (5) is simplified into

$$
F(r)=\xi n p / r^{2},
$$

where $\xi$ is a constant.

In a previous study Equation (6) was called the law of gravity between two clusters, at distance $r$ containing $n$ respectively $p$ oscillators $_{69.9}$ [2]. Note that the cluster can be few oscillators $_{69.9}$, an electron, an atom, a mass, a planet or a black hole.

Equation (6) can be illustrated with gravity between the earth and the sun. The earth contains $n$ atomic oscillators $_{69.9}$ creating a force $n \delta F(r)$ on every atomic oscillator ${ }_{69.9}$ in the sun. The sun contains $p$ oscillators 69.9 , thus the total force of attraction is $n p \delta F(r)$ and that is equal to Equation (6). The earth's mass $m_{1}$ is proportional to the number of atomic oscillators 69.9 on earth, i.e. $m_{1} \sim n$, and the sun's mass $m_{2} \sim p$. Equation (6) is then approximately equal to the Newtonian geometric law of gravity, where $F_{G}$ is the gravitational force:

$$
F_{G}=\xi n p / r^{2} \approx F_{\text {Newton }}=G m_{1} m_{2} / r^{2} .
$$

$S_{1}(r)$ and $S_{2}(r)$ in Equation (6) depend on the distance between the singularity and $S_{1}(r)$ respectively $S_{2}(r)$. Thus $\xi$ in Equation (7) increases at decreasing distance to the singularity.

Gravity is illustrated in Figure 1.

Comment; Gravity encompasses altogether four oscillators at $69.9 \mathrm{~Hz}, 71.9 \mathrm{~Hz} .89 .9 \mathrm{~Hz}$ and $91.9 \mathrm{~Hz}$. Together they form clusters and the sum of $F(r)$ in Equation (6) of all four forces comprises, according to the superposition principle, a static force. This explains why gravity is a static force. This will be explained in detail in a forthcoming study. In this study only the contribution from $69.9 \mathrm{~Hz}$ is described in order to simplify. In the text $69.9 \mathrm{~Hz}$ can be substituted with $69.9 \mathrm{~Hz}, 71.9 \mathrm{~Hz} .89 .9 \mathrm{~Hz}$ and $91.9 \mathrm{~Hz}$ in order to gain a correct description.

\subsection{Photon}

The amplitude of light and radio waves decreases linearly with the distance $r$ from the source (as $1 / r$ ). One commonly known phenomenon in physics which decreases linearly with distance is the far field from accelerated charge, i.e. the plane TEM wave according to Maxwell's equations [5] [6]:

$$
\begin{aligned}
& \operatorname{curl} \mathbf{H}=i+\partial \mathbf{D} / \partial t, \\
& \operatorname{curl} \mathbf{E}=-\partial \mathbf{B} / \partial t .
\end{aligned}
$$

According to state of the art science radio waves are generated by accelerated electrons in the antenna which produces a far field according to Equations (8) and (9). This far field is a plane TEM wave. This TEM wave can be absorbed (e.g. in matter or receiver antenna) whereby its energy is dissipated as e.g. heat or current in the 


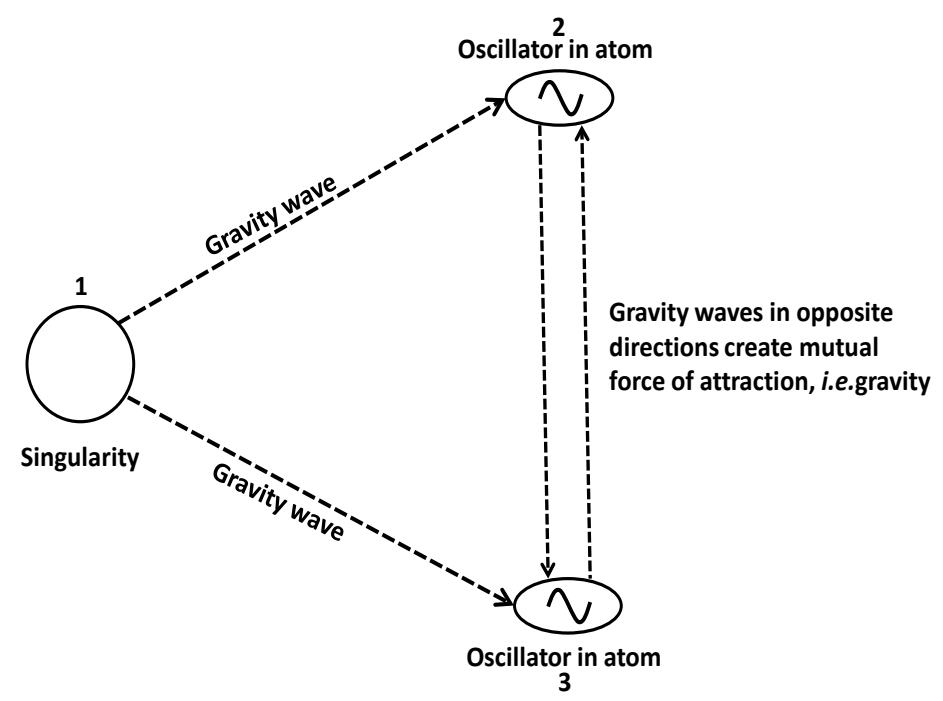

Figure 1. A singularity $\underline{1}$ generates gravity waves (low frequency plane TEM waves). Oscillators ${ }_{69.9} \underline{2}$ and $\underline{3}$ in atoms create resonance with gravity waves and re-emit them unaltered. This causes flow of gravity waves between atoms and their oscillators ${ }_{69.9} \underline{2}$ and $\underline{3}$. The flow of gravity waves in opposite directions between oscillators 69.9 results in mutual force of attraction, i.e. gravity.

antenna and which results in that the TEM wave ceases to exist.

The real process is very different; however, the end result is almost the same. The theory of photon energy transport in space can be illustrated with radio waves. It has been shown [3] that radio waves are the result of the sum of time limited processes, i.e. acceleration or deceleration of oscillators 69.9 in an electron or atomic nucleus, see Figure 2. These oscillators 69.9 absorb and re-emit gravity waves with frequency $69.9 \mathrm{~Hz}$. Accelerating or decelerating an electron or an atomic nucleus implies that they gain energy, i.e. each of their intrinsic oscillators $_{69.9}$ increases its energy and this excess energy can be described by one or many energy quanta $h v$. In this case the oscillator $69.9 \underline{4}$ in the electron or atom contains one energy quantum $h v$ at a time, where $h$ is Planck constant or quantum of action. The energy quantum $h v$ is discrete and limited in time. The energy quantum $h v$ is superpositioned on a re-emitted TEM wave. This TEM wave contains the superpositioned energy quantum $h v$ and the frequency $69.9 \mathrm{~Hz}$ and instant frequency $v$. Hence, the re-emitted wave is a gravity wave with superpositioned electromagnetic energy. The superpositioned electromagnetic energy quantum $h v$ with frequency $v$ is the photon. In a continuous process the sum of many photons and their energy quanta $h v$, superpositioned on re-emitted gravity waves, result in radio waves. The radio wave's amplitude is proportional to the number of photons per time unit, which is proportional to the number of involved energy quanta $h v$, which in its turn is proportional to the number of involved oscillators ${ }_{69.9}$ and which is proportional to the inertial mass. This radio wave has the frequency $69.9 \mathrm{~Hz}+v$.

The radio wave can be absorbed in matter $\underline{5}$ and where the superpositioned energy quanta $h v$ are absorbed, resulting in that the radio wave is converted back to the original gravity wave with frequency $69.9 \mathrm{~Hz}$.

The energy contained in a TEM wave is proportional to its frequency $v$. From this follows that the superpositioned energy and the photon energy $\delta E$ is:

$$
\delta E=\hbar \omega=h \nu .
$$

The TEM wave's superpositioned energy and momentum are related as $\delta E=p c$, where $p$ is the magnitude of the momentum vector $\mathbf{p}$. This derives from the following relativistic relation, with $m=0$ [11]:

$$
\begin{gathered}
\delta E^{2}=p^{2} c^{2}+m^{2} c^{4}, \\
\mathbf{p}=\hbar \mathbf{k},
\end{gathered}
$$

where $\mathbf{k}$ is the wave vector (where the wave number $k=|k|=2 \pi v / c$ ), and $\hbar=h / 2 \pi$ is the reduced Planck 


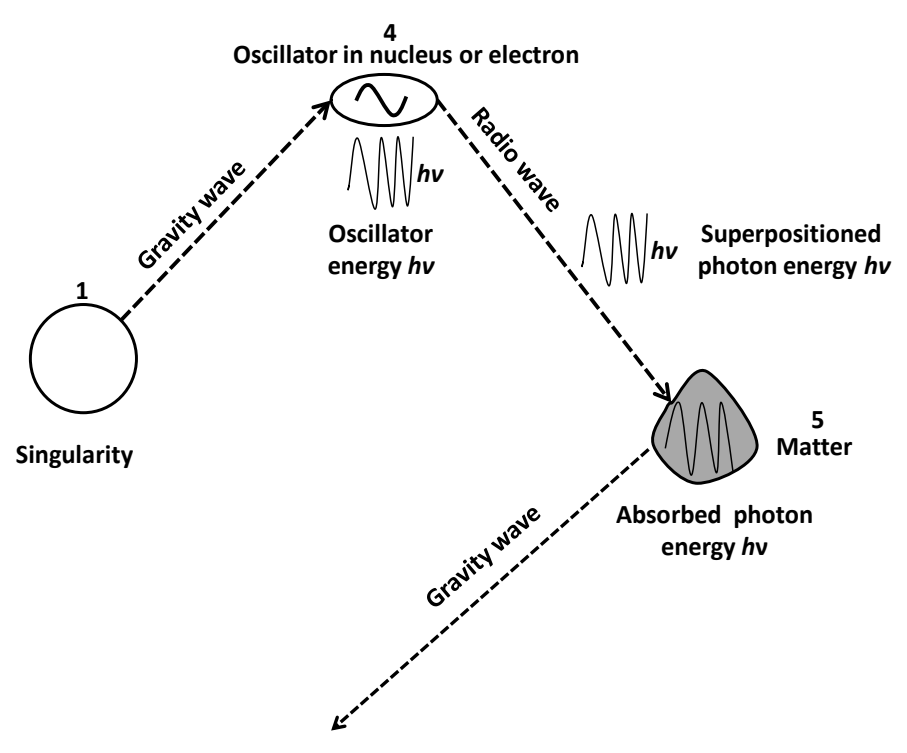

Figure 2. Electrons can be accelerated or decelerated. The electron contains oscillators 69.9 . The oscillator $69.9 \underline{4}$ in an electron creates resonance with a gravity wave and excess energy $h v$, caused by electron acceleration deceleration, is superpositioned on the re-emitted wave. The superpositioned energy $h v$ with frequency $v$ represents the photon. This wave with frequency $69.9 \mathrm{~Hz}$ and $v$ is re-emitted as a radio wave. The superpositioned energy $h v$ can be absorbed in matter $\underline{5}$, whereby the radio wave is converted into the original gravity wave with frequency $69.9 \mathrm{~Hz}$.

constant. Since $\mathbf{p}$ points in the direction of the TEM wave propagation, the magnitude of the momentum is:

$$
p=\hbar k=h v / c \text {. }
$$

Thus, the photon contained in the radio wave is not a particle; however, discrete electromagnetic energy quantum $h v$, with frequency $v$, superpositioned on a low frequency TEM wave, i.e. a gravity wave. From this follows that the photon is strictly mass less, has zero charge and propagates with the speed of the low frequency TEM wave, i.e. with the speed of light in free space.

Previous reports have shown that this process is frequency independent. Currents in the magnetosphere generate photons or radio waves with the period 24 hours [4]. It is possible to generate photons or radio waves in the $2 \mathrm{~Hz}-2 \mathrm{GHz}$ range using a signal generator or a radio transmitter [3]. Photons in the 400 to 700 nanometer range are produced by a similar process [3] when matter is heated. Hence, the theoretical model is valid from almost infinite period to almost infinite frequency.

\subsection{Gravitational Mass}

From Equation (5) follows that the gravitational force $F_{G}$ acting on an oscillator 69.9 or a cluster of $n$ oscillators ${ }_{69.9}$ (e.g. oscillators 69.9 contained in electron, atom, mass, planet) is the result of TEM waves operating on the oscillator $_{69.9}$ or the cluster of $n$ oscillators 69.9 . Hence, the force $F_{G}$ is proportional to the sum of involved oscillators $_{69.9}$.

$$
F_{G}=\zeta \sum_{n} \text { oscillator }_{69.9} .
$$

This implies that the gravitational mass $m_{G}$ is the expression of the mechanism which results in the gravitational force $F_{G}$ :

$$
m_{G}=\alpha \sum_{n} \text { oscillator }_{69.9}
$$

where $\alpha$ is a constant. 
This also implies that Einstein's $E=m c^{2}$ is incorrect. Absorbed TEM waves with frequency $69.9 \mathrm{~Hz}$ do not result in nucleus energy. It is proposed that energy $E$, stored in a body, is the result of TEM waves absorbed by atomic oscillators (with natural frequencies in another frequency range), reported previously [1]. However, the number of these atomic oscillators is proportional to the number of atomic oscillators ${ }_{69.9}$ with natural frequency $69.9 \mathrm{~Hz}$, and proportional to the mass $m_{G}$. Hence, $E=m c^{2}$ is correct in a wide sense.

\subsection{Inertial Mass}

The implication of Section 2.2 is as follows. Accelerating or decelerating an oscillator ${ }_{69.9}$ or a cluster of $n$ oscillators $_{69.9}$ (e.g. particle, electron, nucleus, atom, body, mass, car or planet) results in emitted photons, i.e. emitted electromagnetic energy. Hence, the force $F_{I}$ and the energy $E_{I}$ must be enforced on the oscillator ${ }_{69.9}$ or $n$ oscillators $_{69.9}$ in order achieve acceleration or deceleration. The enforced energy $E_{I}$ equals the sum of the energy in emitted photons. Thus, enforced energy is transformed into emitted electromagnetic energy.

The force $\delta F_{I}$ must be enforced on one oscillator ${ }_{69.9}$ in order to achieve the acceleration or deceleration $\beta \cdot a$, where $\beta$ is a constant and $a$ is the acceleration. In order to accelerate or decelerate $n$ oscillators $_{69.9}$ the force $F_{I}$ must be enforced on the oscillators 69.9 :

$$
F_{I}=\beta a \sum_{n} \text { oscillator }_{69.9} .
$$

In classical mechanics Equation (16) is denoted $F_{I}=m_{I} a$. Consequently, the inertial mass $m_{I}$ is the expression of the mechanism which transforms enforced energy into emitted electromagnetic energy:

$$
m_{I}=\beta \sum_{n} \text { oscillator }_{69.9} \text {. }
$$

From Equation (15) and Equation (17) follows that the gravitational mass relates to the inertial mass; $m_{G}=\alpha / \beta \cdot m_{I}$. This explains the linear relationship between gravitational and inertial mass, as observed in classical mechanics.

Inertial mass can be illustrated with the following. Enforced force $F_{I}$ and energy $E_{I}$ accelerates the mass $m_{I}$, resulting in that photons are emitted and where the photon energy equals $E_{I}$. When $F_{I}$ is removed the mass $m_{I}$ propagates with constant velocity in free space. This implies that the mass does not emit photons, i.e. the transport is loss free. Now the force $F_{I}$ and energy $E_{I}$ is enforced on the mass $m_{I}$, which results in that the mass is decelerated whereby photons are emitted, and where the photon energy equals $E_{I}$. In classical mechanics the kinetic energy is $E_{K}=(1 / 2) m_{I} v^{2}$. However, this energy is not stored in the mass, i.e. the kinetic energy $E_{K}$ does not exist. $E_{K}$ expresses that a mass $m_{I}$ with velocity $\mathbf{v}$ can be decelerated to the velocity $\mathbf{v}=0$ in a given reference system. This results in that photons are emitted and where the total photon energy is

$$
E_{I}=(1 / 2) m_{I} v^{2}=(1 / 2) v^{2} \beta \sum_{n} \text { oscillator }_{69.9} \text {. }
$$

Consequently, accelerating mass results in that enforced energy $E_{I}$ is emitted as photon energy $E_{I}$, and it is not stored as kinetic energy $E_{K}$. Mass deceleration results in that that the enforced energy $E_{I}$ is emitted as photon energy $E_{I}$, and there is no other energy involved, i.e. kinetic energy.

\section{Alternative Theory}

The Higgs field, the Higgs mechanism and the Higgs particle provide an alternative description of inertial mass. The drawback with that theory is that the Higgs field and the Higgs mechanism are based entirely on the theoretical mathematical description of a scalar field which we do not know if it exists, nor has this field and mechanism been measured and empirically verified. The existence of the Higgs particle has been reported [21].

The strength of the present theory is that there exists a method to measure relevant parameters [1]-[4]. TEM waves originating from the singularity have been measured to a degree which allows exact description of their characteristics such as direction of origin, amplitude, phase, frequencies and field vector behavior [1]-[3]. Furthermore, TEM waves, absorbed and re-emitted by atoms, have been measured to a degree which reveals the atomic intrinsic mechanism; forced damped oscillators with natural frequencies $0.005-0.03 \mathrm{~Hz}$ [1] and $69.9 \mathrm{~Hz}$ [2]. Moreover, extremely low frequency photons have been produced and measured in laboratory [3]. Hence, crucial parts of the present theory have been measured in laboratory and some parts have been simulated and reproduced in laboratory. 


\section{Discussion and Conclusion}

It is proposed that all energies in the universe originate from a singularity, and are distributed throughout the universe by low frequency TEM waves, having a broad spectrum of frequencies. The atom in itself is fully passive. At this stage the atom's physical view is of little interest. However, the atom's functional view is crucial; the atom consists of forced damped oscillators with different natural frequencies. They absorb TEM waves originating from the singularity. The atomic oscillators are assembled in different configurations, where the number of oscillators and their mix of natural frequencies result in different types of electromagnetic energies and forces, according to electromagnetic theory [5] [6] and wave theory [7]-[11]. One configuration results in that the absorbed energy creates internal forces and nuclear binding energy [1]. A second configuration results in that the absorbed energy generates what is perceived as static electric fields, e.g. the Coulomb field [1]. A third configuration results in that absorbed energy is re-emitted creating gravity, photon, gravitational mass and inertial mass [2] [3]. All of these processes are entirely electromagnetic. These processes originate from external electromagnetic energy operating on fully passive atoms and where the atom contains only one type of functionality, and force damped oscillators.

The platform of modern physics is elementary particle physics described in the Standard Model [12]. Atom, photon and graviton are described as particles. The atom is self contained, i.e. atomic forces and energy are the consequence of atomic intrinsic mechanisms. The Higgs field is added in order to account for inertial mass.

Consequently, there is a fundamental difference between the present theoretical model and the Standard Model and they provide fundamentally different solutions to the basic level of physics.

The present paper describes that gravitational mass $m_{G}$ is the expression of the mechanism which results in gravitational force $F_{G}$. Inertial mass $m_{I}$ is the expression of the mechanism which transforms enforced energy $E_{I}$ into emitted electromagnetic energy $E_{I}$. In both cases the mechanism consists of external energy operating on atomic oscillators 69.9 with natural frequency $69.9 \mathrm{~Hz}$. The present study describes gravity and photon by their electromagnetic processes. The present study also displays that gravitational and inertial mass are caused by electromagnetic processes.

\section{References}

[1] Giertz, H.W. (2013) Atoms Absorb Low Frequency Electromagnetic Energy. Open Journal of Microphysics, 3, 115120.

[2] Giertz, H.W. (2013) Gravity Caused by TEM Waves Operating on Dipoles in Atoms. International Journal of Astronomy and Astrophysics, 3, 39-50.

[3] Giertz, H.W. (2013) Photons Are EM Energy Superpositioned on TEM Waves. Open Journal of Microphysics, 3, 7180. http://dx.doi.org/10.4236/ojm.2013.33013

[4] Giertz, H.W. (2010) Extremely Low Frequency Electromagnetic Energy in the Air. Journal of Atmospheric and Solar-Terrestrial Physics, 72, 767-773. http://dx.doi.org/10.1016/j.jastp.2010.03.022

[5] Melrose, D.B. and McPhedran, R.C. (1991) Electromagnetic Processes in Dispersive Media. Cambridge University Press, Cambridge. http://dx.doi.org/10.1017/CBO9780511600036

[6] Bleaney, B.I. and Bleaney, B. (1965) Electricity and Magnetism. Oxford University Press, London.

[7] Kneubühl, F.K. (1997) Oscillations and Waves. Springer, Berlin. http://dx.doi.org/10.1007/978-3-662-03468-2

[8] Serway, R.A. and Jewett, J.W. (2005) Principles of Physics. 4th Edition, Cengage Learning, Stamford.

[9] Ostrovsky, L.A. and Potapov, A.I. (2002) Modulated Waves: Theory and Application. Johns Hopkins University Press, Baltimore.

[10] Terras, A. (1999) Fourier Analysis on Finite Groups and Applications. Cambridge University Press, Cambridge.

[11] Alonso, M. and Finn, E.J. (1968) Fundamental University Physics Volume III: Quantum and Statistical Physics. Addison-Wesley, Boston.

[12] Burgess, C. and Moore, G. (2007) The Standard Model: A Primer. Cambridge University Press, Cambridge.

[13] Bialynicki-Birula, I. (1994) On the Wave Function of the Photon. Acta Physica Polonica A, 86, 97-116.

[14] Sipe, J.E. (1995) Photon Wave Functions. Physical Review A, 52, 1875-1883.

http://dx.doi.org/10.1103/PhysRevA.52.1875

[15] Higgs, P.W. (1964) Broken Symmetries, Massles Particles and Gauge Fields. Physics Letters, 12, 132-133. http://dx.doi.org/10.1016/0031-9163(64)91136-9 
[16] Higgs, P.W. (1964) Broken Symmetries and the Masses of Gauge Bosons. Physical Review Letters, 13, 508-509. http://dx.doi.org/10.1103/PhysRevLett.13.508

[17] Englert, F. and Brout, R. (1964) Broken Symmetry and the Mass of Gauge Vector Mesons. Physical Review Letters, 13, 321-323. http://dx.doi.org/10.1103/PhysRevLett.13.321

[18] Bezrukov, F. and Shaposhnikov, M. (2008) The Standard Model Higgs Boson as the Inflation. Physics Letters B, 659, 703-706. http://dx.doi.org/10.1016/j.physletb.2007.11.072

[19] Oda, I. (2010) Remarks on Higgs Mechanism for Gravitons. Physics Letters B, 690, 322-327. http://dx.doi.org/10.1016/j.physletb.2010.05.048

[20] De Palma, G. and Strocchi, F. (2013) A Non-Perturbative Argument for the Non-Abelian Higgs Mechanism. Annals of Physics, 336, 112-117. http://dx.doi.org/10.1016/j.aop.2013.05.012

[21] ATLAS Collaboration (2013) Measurement of Higgs Boson Production and Couplings in Diboson Final States with the ATLAS Detector at the LHC. Physics Letters B, 726, 88-119. http://dx.doi.org/10.1016/j.physletb.2013.08.010. 